\title{
Preparation of Cyclophanes by Room-Temperature Ring-Closing Alkyne Metathesis with Imidazolin-2-iminato Tungsten Alkylidyne Complexes
}

Stephan Beer, Kai Brandhorst, Jörg Grunenberg, Cristian Hrib, Peter G. Jones and Matthias Tamm*

Institut für Anorganische und Analytische Chemie, Technische Universität Carolo-Wilhelmina, Hagenring 30, D-38106 Braunschweig, Germany

\section{Supporting Information}

Index

I. General

II. Preparation of starting materials

III. Ring-closing alkyne metathesis

IV. Crystal Structure Determinations

V. Mathematical details for the calculation of the monomer-/dimer- ratio from computed Gibbs free energy changes

VI. Electronic Structure Calculations

VII. Cartesian coordinates of the minimum energy structures of the ortho-, meta- and paramonomers and dimers $(\mathrm{B} 3 \mathrm{LYP} / 6-311 \mathrm{G}(\mathrm{d}, \mathrm{p})$

VIII. Spectra 


\section{General}

All operations with air and moisture-sensitive compounds were performed in a glove box under a dry argon atmosphere (MBraun 200B) or on a high vacuum line using Schlenk techniques. All solvents were purified by a solvent purification system from MBraun and stored over molecular sieve (4 $\AA$ ) prior to use. The ${ }^{1} \mathrm{H}$ and ${ }^{13} \mathrm{C}$ spectra were recorded on Bruker DPX $200(200 \mathrm{MHz})$, Bruker DRX $400(400 \mathrm{MHz})$, and Bruker DPX $600(600 \mathrm{MHz})$, devices. The chemical shifts are expressed in parts per million (ppm) using tetramethylsilane (TMS) as internal standard. Coupling constants $(J)$ are reported in Hertz $(\mathrm{Hz})$, and splitting patterns are indicated as $\mathrm{s}$ (singlet), d (doublet), t (triplet), q (quartet), m (multiplet) and br (broad). Elemental analysis (C, $\mathrm{H}$, N) succeeded by combustion and gas chromatographical analysis with an Elementar varioMICRO. GC analysis was performed on a SHIMADZU GC-2010, GC-MS were accomplished on a JEOL AccuTOF, MS on a Finnigan MAT 95 (EI), Finnigan MAT 95 XL (ESI), respectively and HR-MS from Bruker-Demo QTOF micro. Unless otherwise indicated, all starting materials were obtained from Aldrich and were used without further purification. Flash chromatography was performed using Merck silica gel (230-400 mesh).

\section{Preparation of starting materials}

Catalyst $\mathbf{1}^{1}$ was prepared following literature procedures.

\section{General synthesis of the diynes (Williamson etherification):}

In a $250 \mathrm{ml}$ Schlenk flask $0.060 \mathrm{~mol}$ of 3-pentyn-1-ol were dissolved in $30 \mathrm{ml}$ THF and degassed several times. $0.040 \mathrm{~mol}$ sodium were added and stirred for $2 \mathrm{~h}$ at room temperature. A solution of $0.015 \mathrm{~mol}$ of the dihalide in $25 \mathrm{ml}$ THF were added to the resulting sodium salt and refluxed over night. After cooling the suspension to room temperature the reaction was quenched by adding $100 \mathrm{ml} \mathrm{H}_{2} \mathrm{O}$. The product was extracted with $60 \mathrm{ml} \mathrm{Et}_{2} \mathrm{O}$ and washed several times with $25 \mathrm{ml} \mathrm{H}{ }_{2} \mathrm{O}$. Evaporation of the solvent afforded the pure product in yields $>68 \%$ as light yellow oils or white solids, respectively.

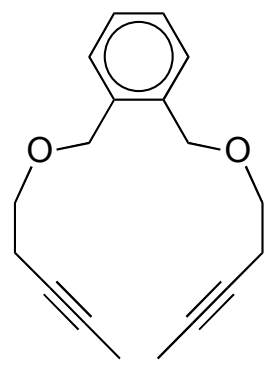

$2 \mathbf{a}$

\section{o-Xylylenedi(oxapent-3-yne)}

${ }^{1}$ Beer, S.; Hrib, C. G.; Jones, P. G.; Brandhorst, K.; Grunenberg, J.; Tamm, M. Angew. Chem. 2007, 119, 9047; Angew. Chem;. Int. Ed. 2007, 46, 8890. 
Isolated yield: $89 \%$ as colorless oil

${ }^{1} \mathrm{H}$ NMR $\left(200.1 \mathrm{MHz}, \mathrm{CDCl}_{3}, 25{ }^{\circ} \mathrm{C}\right): \delta 1.78\left(6 \mathrm{H}, \mathrm{t}, \mathrm{CH}_{3}\right), 2.43\left(4 \mathrm{H}, \mathrm{tq}, \mathrm{CCH}_{2}\right), 3.56(4 \mathrm{H}, \mathrm{t}$, $\left.\mathrm{CH}_{2}\right), 4.63\left(4 \mathrm{H}, \mathrm{s}, \mathrm{OCH}_{2}\right), 7.31(4 \mathrm{H}, \mathrm{m}, \mathrm{Ar} H) ;{ }^{13} \mathrm{C} \mathrm{NMR}\left(50.3 \mathrm{MHz}, \mathrm{CDCl}_{3}, 25{ }^{\circ} \mathrm{C}\right): \delta 3.5(\mathrm{~s}$, $\left.\mathrm{CH}_{3}\right), 20.1\left(\mathrm{~s}, \mathrm{CCH}_{2}\right), 69.0\left(\mathrm{~s}, \mathrm{OCH}_{2}\right), 70.6\left(\mathrm{~s}, \mathrm{OCH}_{2}\right), 75.9(\mathrm{~s}, C \equiv C), 76.8(\mathrm{~s}, C \equiv C), 127.8(\mathrm{~s}, \mathrm{Ar}-$ $C), 128.7$ (s, Ar-C), 136.4 (s, Ar-C); elemental analysis: calculated for $\mathrm{C}_{18} \mathrm{H}_{22} \mathrm{O}_{2}$ : C $79.96 \%, \mathrm{H}$ $8.20 \%$; found: C $79.97 \%$, H $8.03 \%$.

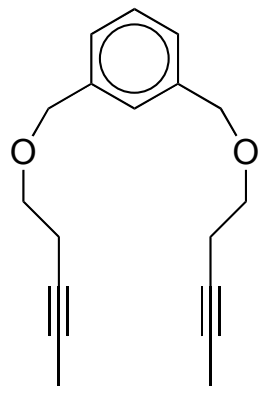

2b

\section{m-Xylylenedi(oxapent-3-yne)}

Isolated yield: $91 \%$ as yellow oil

${ }^{1} \mathrm{H}$ NMR $\left(200.1 \mathrm{MHz}, \mathrm{CDCl}_{3}, 25{ }^{\circ} \mathrm{C}\right): \delta 1.77\left(6 \mathrm{H}, \mathrm{t},{ }^{5} \mathrm{~J}_{\mathrm{HH}}=2.6 \mathrm{~Hz}, \mathrm{CH}_{3}\right), 2.44\left(4 \mathrm{H}, \mathrm{tq}, \mathrm{CCH}_{2}\right)$, $3.54\left(4 \mathrm{H}, \mathrm{t},{ }^{3} \mathrm{~J}_{\mathrm{HH}}=7.1 \mathrm{~Hz} \mathrm{CH}\right), 4.54\left(4 \mathrm{H}, \mathrm{s}, \mathrm{OCH}_{2}\right), 7.28(4 \mathrm{H}, \mathrm{m}, \mathrm{ArH}) ;{ }^{13} \mathrm{C} \mathrm{NMR}(50.3 \mathrm{MHz}$, $\left.\mathrm{CDCl}_{3}, 25{ }^{\circ} \mathrm{C}\right): \delta 4.6\left(\mathrm{~s}, \mathrm{CH}_{3}\right), 21.3\left(\mathrm{~s}, \mathrm{CCH}_{2}\right), 70.0\left(\mathrm{~s}, \mathrm{OCH}_{2}\right), 73.9\left(\mathrm{~s}, \mathrm{OCH}_{2}\right), 77.0(\mathrm{~s}, C \equiv C)$, $77.8(\mathrm{~s}, C \equiv C), 128.1(\mathrm{~s}, \operatorname{Ar}-C), 129.6$ (s, Ar-C), 139.6 (s, Ar-C); elemental analysis: calculated for $\mathrm{C}_{18} \mathrm{H}_{22} \mathrm{O}_{2}$ : C $79.96 \%$, H $8.20 \%$; found: C $79.57 \%$, H $8.26 \%$.

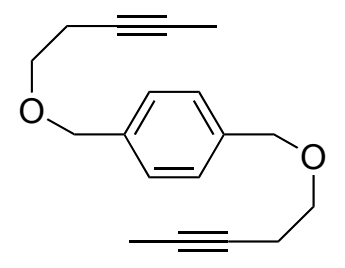

2c

\section{p-Xylylenedi(oxapent-3-yne)}

Isolated yield: $68 \%$ as white solid

${ }^{1} \mathrm{H}$ NMR $\left(200.1 \mathrm{MHz}, \mathrm{CDCl}_{3}, 25^{\circ} \mathrm{C}\right): \delta 1.78\left(6 \mathrm{H}, \mathrm{t}, \mathrm{CH}_{3}\right), 2.43\left(4 \mathrm{H}, \mathrm{tq}, \mathrm{CCH}_{2}\right), 3.54(4 \mathrm{H}, \mathrm{t}$, $\left.\mathrm{CH}_{2}\right), 4.54\left(4 \mathrm{H}, \mathrm{s}, \mathrm{OCH}_{2}\right), 7.32(4 \mathrm{H}, \mathrm{s}, \mathrm{Ar} H) ;{ }^{13} \mathrm{C} \mathrm{NMR}\left(50.3 \mathrm{MHz}, \mathrm{CDCl}_{3}, 25{ }^{\circ} \mathrm{C}\right): \delta 3.5(\mathrm{~s}$, $\left.\mathrm{CH}_{3}\right), 20.1\left(\mathrm{~s}, \mathrm{CCH}_{2}\right), 68.7\left(\mathrm{~s}, \mathrm{OCH}_{2}\right), 72.6\left(\mathrm{~s}, \mathrm{OCH}_{2}\right), 75.8(\mathrm{~s}, C \equiv C), 77.4(\mathrm{~s}, C \equiv C), 127.8(\mathrm{~s}, \mathrm{Ar}-$ $C$ ), 137.6 (s, Ar-C); elemental analysis: calculated for $\mathrm{C}_{18} \mathrm{H}_{22} \mathrm{O}_{2}$ : C $79.96 \%, \mathrm{H} 8.20 \%$; found: C 79.94 \%, H $8.40 \%$. 


\section{Ring closing alkyne metathesis (RCAM)}

A $4.5 \mathrm{mM}$ hexane solution of the diynes $2(150.0 \mathrm{mg}, 0.055 \mathrm{mmol})$ and catalyst $1(9.0 \mathrm{mg}$, $1.11 \cdot 10^{-5} \mathrm{~mol}, 2 \mathrm{~mol} \%$ ) was stirred at room temperature under reduced pressure (350 mbar). After $2 \mathrm{~h}$ the solution was filtered through alumina in order to remove the catalyst 1 and elution with $\mathrm{Et}_{2} \mathrm{O}$ afforded the cyclization products after evaporation of the solvent. In the case of $\mathbf{3 b}$ and $4 c$ colorless crystals could be obtained from a cooled $\left(4{ }^{\circ} \mathrm{C}\right)$ hexane or $\mathrm{CHCl}_{3}$ solution, respectively. Employing 2a afforded a mixture of monomeric cycloalkyne 3a and dimeric cycloalkyne $4 \mathbf{a}$ in a 24:76 ratio according to a ${ }^{1} \mathrm{H}$ NMR spectrum, which was taken from the crude product after evaporation of the solvent. Isolation of $\mathbf{3 a}$ and $4 \mathbf{a}$ could be achieved by fractional crystallization from hexane, whereby the dimeric product precipitated. After removal of $4 \mathbf{a}$ colorless crystals of $\mathbf{3 a}$ could be obtained from the supernatant hexane solution.

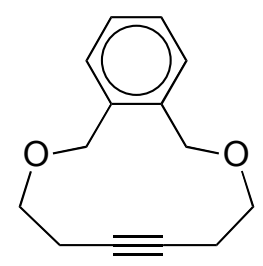

3a

\section{2,9-Dioxa[10]orthocyclophane-5-yne}

Isolated yield: $18 \%$ as colorless crystals

${ }^{1} \mathrm{H}$ NMR $\left(200.1 \mathrm{MHz}, \mathrm{CDCl}_{3}, 25{ }^{\circ} \mathrm{C}\right): \delta 2.41\left(4 \mathrm{H}, \mathrm{tt}, \mathrm{C} \equiv \mathrm{CCH}_{2}\right), 3.73\left(4 \mathrm{H}, \mathrm{t}, \mathrm{OCH}_{2}\right), 4.87(4 \mathrm{H}$, $\left.\mathrm{s}, \mathrm{OCH}_{2}\right), 7.33(4 \mathrm{H}, \mathrm{m}, \mathrm{ArH}) .{ }^{13} \mathrm{C} \mathrm{NMR}\left(50.3 \mathrm{MHz}, \mathrm{CDCl}_{3}, 25{ }^{\circ} \mathrm{C}\right): \delta 21.2\left(\mathrm{~s}, \mathrm{CH}_{2}\right), 68.4(\mathrm{~s}$, $\left.\mathrm{OCH}_{2}\right), 71.7\left(\mathrm{~s}, \mathrm{OCH}_{2}\right), 80.9(\mathrm{~s}, C \equiv C), 128.1$ (s, Ar-C), 130.1 (s, Ar-C), 138.5 (s, ipso-C). elemental analysis: calculated for $\mathrm{C}_{14} \mathrm{H}_{16} \mathrm{O}_{2}$ : C $77.75 \%, \mathrm{H} 7.46 \%$; found: $\mathrm{C} 77.54 \%, \mathrm{H} 7.66 \%$. MS (ESI), m/z (\% relative intensity): $239.1\left(\mathrm{M}^{+}+\mathrm{Na}, 100\right)$. GC-MS (EI), m/z (relative intensity): 215 (M+, 10); 197 (15); 171 (100); 155 (18); 141 (20); 119 (19); 104 (19); 91 (21); 77 (24); 65 (8).

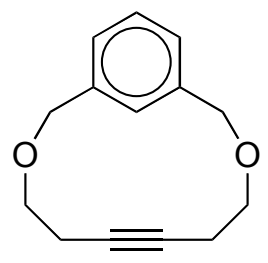

$3 \mathbf{b}$

\section{2,9-Dioxa[10]metacyclophane-5-yne}

Isolated yield: $93 \%$ as colorless crystals

${ }^{1} \mathrm{H}$ NMR $\left(200.1 \mathrm{MHz}, \mathrm{CDCl}_{3}, 25{ }^{\circ} \mathrm{C}\right): \delta 2.45\left(4 \mathrm{H}, \mathrm{tt},{ }^{3} \mathrm{~J}_{\mathrm{HH}}=5.2 \mathrm{~Hz}, \mathrm{C} \equiv \mathrm{CCH}_{2}\right), 3.65\left(4 \mathrm{H}, \mathrm{t},{ }^{3} \mathrm{~J}_{\mathrm{HH}}\right.$ $\left.=4.8 \mathrm{~Hz}, \mathrm{OCH}_{2}\right), 4.65\left(4 \mathrm{H}, \mathrm{s}, \mathrm{OCH}_{2}\right), 7.03(2 \mathrm{H}, \mathrm{d}(\mathrm{q}), \operatorname{Ar} H), 7.27(1 \mathrm{H}, \mathrm{m}, \mathrm{ArH}), 8.30(1 \mathrm{H}, \mathrm{s}(\mathrm{q})$, 
$\mathrm{ArH}) .{ }^{13} \mathrm{C}$ NMR $\left(50.3 \mathrm{MHz}, \mathrm{CDCl}_{3}, 25^{\circ} \mathrm{C}\right): \delta 20.9\left(\mathrm{~s}, \mathrm{CH}_{2}\right), 68.9\left(\mathrm{~s}, \mathrm{OCH}_{2}\right), 71.1\left(\mathrm{~s}, \mathrm{OCH}_{2}\right)$, 79.5 (s, $C \equiv C$ ), 124.5 (s, $\operatorname{Ar}-C$ ), 125.0 (s, $\operatorname{Ar}-C$ ), 127.6 (s, $\operatorname{Ar}-C$ ), 139.7 (s, ipso-C). elemental analysis: calculated for $\mathrm{C}_{14} \mathrm{H}_{16} \mathrm{O}_{2}$ : $\mathrm{C} 77.75 \%, \mathrm{H} 7.46 \%$; found: $\mathrm{C} 77.66 \%, \mathrm{H} 7.70 \%$.

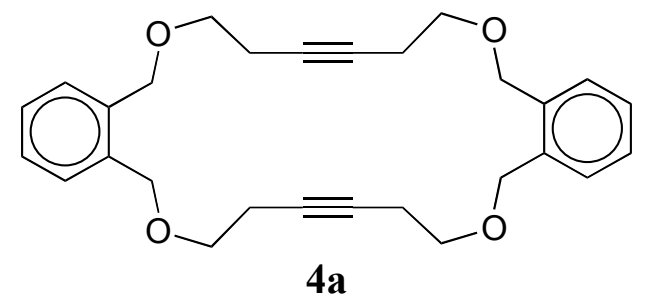

\section{2,9,2',9'-Tetraoxa[10.10]orthocyclophane-5,5'-diyne}

Isolated yield: $65 \%$ as white solid

${ }^{1} \mathrm{H}$ NMR $\left(200.1 \mathrm{MHz}, \mathrm{CDCl}_{3}, 25^{\circ} \mathrm{C}\right): \delta 2.50\left(4 \mathrm{H}, \mathrm{tt}, \mathrm{C} \equiv \mathrm{CCH}_{2}\right), 3.62\left(4 \mathrm{H}, \mathrm{t}, \mathrm{OCH}_{2}\right), 4.68(4 \mathrm{H}$, s, $\left.\mathrm{OCH}_{2}\right), 7.33(4 \mathrm{H}, \mathrm{m}, \mathrm{ArH}) .{ }^{13} \mathrm{C}$ NMR $\left(50.3 \mathrm{MHz}, \mathrm{CDCl}_{3}, 25{ }^{\circ} \mathrm{C}\right): \delta 19.6\left(\mathrm{~s}, \mathrm{CH}_{2}\right), 68.5(\mathrm{~s}$, $\mathrm{OCH}_{2}$ ), 70.3 (s, $\mathrm{OCH}_{2}$ ), 77.7 (s, $\left.C \equiv C\right), 127.3$ (s, Ar-C), 128.5 (s, Ar-C), 135.9 (s, ipso-C). elemental analysis: calculated for $\mathrm{C}_{14} \mathrm{H}_{16} \mathrm{O}_{2}$ : C $77.75 \%, \mathrm{H} 7.46 \%$; found: $\mathrm{C} 77.12 \%, \mathrm{H} 7.30 \%$. MS (ESI), $\mathrm{m} / \mathrm{z}$ (\% relative intensity): $456.2\left(\mathrm{M}^{+}+\mathrm{Na}, 100\right)$.

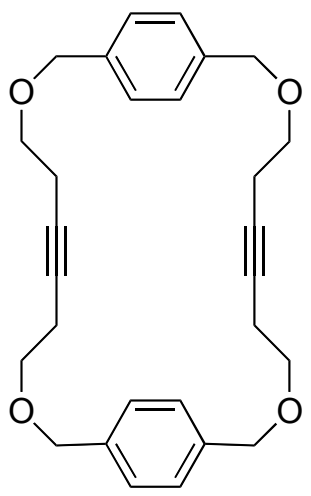

$4 c$

\section{2,9,2',9'-Tetraoxa[10.10]metacyclophane-5,5'-diyne}

Isolated yield: $68 \%$ as colorless crystals

${ }^{1} \mathrm{H}$ NMR $\left(200.1 \mathrm{MHz}, \mathrm{CDCl}_{3}, 25^{\circ} \mathrm{C}\right): \delta 2.45\left(8 \mathrm{H}, \mathrm{tt}, \mathrm{C} \equiv \mathrm{CCH}_{2}\right), 3.51\left(8 \mathrm{H}, \mathrm{t}, \mathrm{OCH}_{2}\right), 4.55(8 \mathrm{H}$, s, $\left.\mathrm{OCH}_{2}\right), 7.28(8 \mathrm{H}, \mathrm{s}, \mathrm{ArH}) ;{ }^{13} \mathrm{C}$ NMR $\left(50.3 \mathrm{MHz}, \mathrm{CDCl}_{3}, 25^{\circ} \mathrm{C}\right): \delta 20.3\left(\mathrm{~s}, \mathrm{CH}_{2}\right), 68.3(\mathrm{~s}$, $\mathrm{OCH}_{2}$ ), $72.6\left(\mathrm{~s}, \mathrm{OCH}_{2}\right), 78.2(\mathrm{~s}, C \equiv C), 127.7$ (s, Ar- $C$ ), 137.7 (s, ipso-C); elemental analysis: calculated for $\mathrm{C}_{14} \mathrm{H}_{16} \mathrm{O}_{2}$ : C $77.75 \%, \mathrm{H} 7.46 \%$; found: C $77.75 \%, \mathrm{H} 8.10 \%$. 


\section{Crystal Structure Determinations}

The hydrogen atoms were included in the model at geometrically calculated positions and refined using a riding model. Diffractometer: Bruker APEX2. Program system: SHELXL-97 (G. M. Sheldrick, University of Göttingen).

Crystal data of $3 \mathbf{b}: \mathrm{C}_{14} \mathrm{H}_{16} \mathrm{O}_{2}, \mathrm{M}_{\mathrm{r}}=216.27 \mathrm{~g} / \mathrm{mol}$, crystal dimensions $0.10 \times 0.05 \times 0.03 \mathrm{~mm}^{3}$, monoclinic, $\mathrm{P} 2{ }_{1} / \mathrm{c}, a=8.292(4), b=16.943(9), c=8.169(4) \AA, \beta=93.057(14)^{\circ}, V=1146.1(10)$ $\AA^{3}, Z=4, \rho_{\text {calc }}=1.253 \mathrm{Mg} / \mathrm{m}^{3}, \mu=0.082 \mathrm{~mm}^{-1}, \operatorname{MoK}_{\alpha}\left(\lambda=0.71073 \mathrm{~nm}^{-1}\right), T=100 \mathrm{~K}, 2 \theta_{\max }=50$, 17243 reflections, 2027 independent $\left(R_{\text {int }}=0.1899\right), R_{1}(I>2 \sigma(I))=0.0583, w R_{2}$ (all data) $=$ $0.1597, \Delta \rho=0.27 /-0.31$ e. $\AA^{-3}$.

Crystal data of $4 \mathrm{c}: \mathrm{C}_{28} \mathrm{H}_{32} \mathrm{O}_{4}, \mathrm{M}_{\mathrm{r}}=432.54 \mathrm{~g} / \mathrm{mol}$, crystal dimensions $0.18 \times 0.10 \times 0.04 \mathrm{~mm}^{3}$, monoclinic, $\mathrm{P} 2{ }_{1} / \mathrm{c}, a=12.9818(8), b=9.8352(7), c=8.9683(6) \AA, \beta=90.586(4)^{\circ}, V=$ $1145.00(13) \AA^{3}, Z=2, \rho_{\text {calc }}=1.255 \mathrm{Mg} / \mathrm{m}^{3}, \mu=0.082 . \mathrm{mm}^{-1}, \operatorname{MoK}_{\alpha}\left(\lambda=0.71073 \mathrm{~nm}^{-1}\right), T=$ $100 \mathrm{~K}, 2 \theta_{\max }=56,15577$ reflections, 2813 independent $\left(R_{\text {int }}=0.0707\right), R_{1}(I>2 \sigma(I))=0.0843$, $w R_{2}($ all data $)=0.2488, \Delta \rho=0.37 /-0.37 \mathrm{e} . \AA^{-3}$.

\section{Mathematical details for the calculation of the monomer/dimer-ratio from computed Gibbs free energies}

The underlying mathematics for the given ratios of the concentrations at the thermodynamic equilibrium of the ortho-, meta-, para- monomer and dimer are as following:

All $\Delta G$ values have been obtained by statistical thermodynamics as implemented in the Gaussian03 set of programs ${ }^{2}$. The equilibrium constants $K$ have been calculated via the van't Hoff relationship between standard free energy change and equilibrium constant $K$

\footnotetext{
${ }^{2}$ M. J. Frisch, G. W. Trucks, H. B. Schlegel, G. E. Scuseria, M. A. Robb, J. R. Cheeseman, J. A. Montgomery, Jr., T. Vreven, K. N. Kudin, J. C. Burant, J. M. Millam, S. S. Iyengar, J. Tomasi, V. Barone, B. Mennucci, M. Cossi, G. Scalmani, N. Rega, G. A. Petersson, H. Nakatsuji, M. Hada, M. Ehara, K. Toyota, R. Fukuda, J. Hasegawa, M. Ishida, T. Nakajima, Y. Honda, O. Kitao, H. Nakai, M. Klene, X. Li, J. E. Knox, H. P. Hratchian, J. B. Cross, V. Bakken, C. Adamo, J. Jaramillo, R. Gomperts, R. E. Stratmann, O. Yazyev, A. J. Austin, R. Cammi, C. Pomelli, J. W. Ochterski, P. Y. Ayala, K. Morokuma, G. A. Voth, P. Salvador, J. J. Dannenberg, V. G. Zakrzewski, S. Dapprich, A. D. Daniels, M. C. Strain, O. Farkas, D. K. Malick, A. D. Rabuck, K. Raghavachari, J. B. Foresman, J. V. Ortiz, Q. Cui, A. G. Baboul, S. Clifford, J. Cioslowski, B. B. Stefanov, G. Liu, A. Liashenko, P. Piskorz, I. Komaromi, R. L. Martin, D. J. Fox, T. Keith, M. A. Al-Laham, C. Y. Peng, A. Nanayakkara, M. Challacombe, P. M. W. Gill, B. Johnson, W. Chen, M. W. Wong, C. Gonzalez, and J. A. Pople, Revision D.02, Gaussian, Inc., Wallingford CT, 2004.
} 


$$
\Delta G=-R T \ln K
$$

where $R$ is the gas constant and $T$ is the temperature in Kelvin. Solving for $K$ results in

$$
K=\exp \left(-\frac{\Delta G}{R T}\right) .
$$

As $K_{c}$ is defined as the ratio between the concentrations at equilibrium of the product and the educt, for the equation

$$
2 m \Leftrightarrow d
$$

we obtain

$$
K_{c}=\frac{c(d)}{c(m)^{2}} \text {. }
$$

If all concentrations have the dimension $\mathrm{mol} \cdot \mathrm{l}^{-1}, K_{c}=K$ and thus

$$
\exp \left(-\frac{\Delta G}{R T}\right)=\frac{c(d)}{c(m)^{2}}
$$

To obtain a ratio of $m$ and $d$ in dependence of the initial concentration of the monomer $c_{0}(m)$ we need the relation

$$
c_{0}(m)=2 \cdot c(d)+c(m)
$$

Solving the above equation (6) for $c(d)$, substitution of that expression into eq. (5) and solving the latter for $c(m)$ finally leads to the expression

$$
c(m)=\frac{\sqrt{1+8 \exp \left(-\frac{\Delta G}{R T}\right) c_{0}(m)}-1}{4 \exp \left(-\frac{\Delta G}{R T}\right)}
$$

By applying eq. (6) again, the concentration of $d$ is given by 


$$
c(d)=\frac{c_{0}(m)}{2}-\frac{\sqrt{1+8 \exp \left(-\frac{\Delta G}{R T}\right) c_{0}(m)}-1}{8 \exp \left(-\frac{\Delta G}{R T}\right)}
$$

The last step is the calculation of the fraction $r_{m}$ of $c(m)$ according to

$$
r_{m}=\frac{c(m)}{c(m)+c(d)}
$$

which for given $\Delta G, R$ and $T$ can then be plotted as a function of $c_{0}(m)$.

\section{Electronic Structure Calculations}

The gas phase global minima of the relevant molecules $\mathbf{3 a}-\mathbf{4 b}$ were obtained by first applying an extended conformational analysis using the MMFF94 force field ${ }^{3}$ together with a Monte Carlo torsional sampling as implemented in the Macromodel 9.5 program $^{4}$. Each lowest energy conformation of $\mathbf{3 a}-\mathbf{4 b}$, respectively, has then been optimized by applying density functional theory. The B3LYP hybrid functional ${ }^{5}$ was employed, and all atoms were described by a standard triple zeta all electron basis set augmented with one set of polarization functions $(6-311 \mathrm{G}(\mathrm{d}, \mathrm{p}))$. After the relevant stationary points were localized on the energy surface, they were further characterized as minima states by normal mode analysis based on the analytical energy second derivatives. Enthalphic and entropic contributions were calculated by statistical thermodynamics as implemented in the Gaussian03 set of programs. ${ }^{2}$

\section{Cartesian coordinates of the minimum energy structures of the ortho-, meta- and para- monomers and dimers $(B 3 L Y P / 6-311 G(d, p)$}

\footnotetext{
${ }^{3}$ Halgren, T. A J. Comp. Chem. 1996, 17, 490-519.

${ }^{4}$ MacroModel version 9.5, Schrödinger, LLC, New York, NY, 2007.

${ }^{5}$ Becke, A. D. J. Chem. Phys. 1993, 98, 5648-5652.
} 


\section{3a (ortho-monomer)}

$\mathrm{E}=-435277,0732771 \mathrm{a} \cdot \mathrm{u}$.

C $-1,466080,60990-0,35109$

C $-1,46608-0,60990 \quad 0,35109$

C $-2,68539-1,20380 \quad 0,67995$

C $-3,89712-0,606250,34041$

C $\quad-3,897120,60623-0,34043$

C $\quad-2,685391,20379-0,67995$

$\mathrm{H} \quad-2,68331-2,14902 \quad 1,21307$

$\mathrm{H} \quad-4,83254-1,08528 \quad 0,60721$

$\mathrm{H} \quad-4,83254 \quad 1,08525-0,60724$

$\mathrm{H} \quad-2,68332 \quad 2,14901-1,21308$

C $\quad-0,18004-1,28675 \quad 0,75780$

$\mathrm{H} \quad 0,53568-0,54724 \quad 1,12535$

$\mathrm{H} \quad-0,38580-2,00048 \quad 1,56662$

C $\quad-0,18004 \quad 1,28676-0,75779$

$\mathrm{H} \quad 0,53568 \quad 0,54725-1,12533$

$\mathrm{H} \quad-0,385792,00048-1,56660$

$\begin{array}{llll}0 & 0,36898 & 1,99092 & 0,36765\end{array}$

$0 \quad 0,36899-1,99091-0,36763$

C $\quad 1,59400 \quad 2,65936 \quad 0,09938$

$\mathrm{H} \quad 1,73998 \quad 2,78388-0,98029$

$\mathrm{H} \quad 1,52618 \quad 3,65610 \quad 0,54784$

C $\quad 2,80661 \quad 1,92867 \quad 0,70847$

$\mathrm{H} \quad 2,687651,904921,79728$

$\mathrm{H} \quad 3,70854 \quad 2,51829 \quad 0,50258$

C $\quad 1,59401-2,65935-0,09935$

$\mathrm{H} \quad 1,74001-2,78382 \quad 0,98032$

$\mathrm{H} \quad 1,52619-3,65610-0,54776$

C $\quad 2,80661-1,92868-0,70848$

$\mathrm{H} \quad 2,68764-1,90497-1,79729$

$\mathrm{H} \quad 3,70855-2,51829-0,50258$

C $\quad 2,9532900,56575 \quad 0,20554$

C $\quad 2,95330-0,56575-0,20560$

\section{4a (ortho-dimer)}

$\mathrm{E}=-870566,7232050$

C $\quad 6,54659-0,55896 \quad 0,78017$

C $\quad 5,41599-0,63933-0,03449$

C $\quad 4,23174-1,19727 \quad 0,48818$

C $\quad 4,21759-1,65300 \quad 1,80592$

C $\quad 5,35563-1,56565 \quad 2,60645$

C $\quad 6,52699-1,01968 \quad 2,09501$

$\mathrm{H} \quad 7,45771-0,12946 \quad 0,37473$

$\mathrm{H} \quad 3,30483-2,08210 \quad 2,19613$

$\mathrm{H} \quad 5,32228-1,92642 \quad 3,62863$
$\mathrm{H}$

C

c

C

$\mathrm{H}$

C

C

$\mathrm{H}$

$\mathrm{H}$

$\mathrm{H}$

C

$\mathrm{H}$

$\mathrm{H}$

C

$\mathrm{H}$

$\mathrm{H}$

C

$\mathrm{H}$

$\mathrm{H}$

C

$\mathrm{H}$

$\mathrm{H}$

O

0

O

O

C

$\mathrm{H}$

$\mathrm{H}$

C

$\mathrm{H}$

$\mathrm{H}$

C

$\mathrm{H}$

$\mathrm{H}$

C

$\mathrm{H}$

$\mathrm{H}$

C

$\mathrm{H}$

$\mathrm{H}$

C

$\mathrm{H}$

$\mathrm{H}$

C
$7,41662-0,94824 \quad 2,71054$

$-5,415990,63933-0,03448$

$\begin{array}{llll}-6,54659 & 0,55896 & 0,78017\end{array}$

$\begin{array}{lll}-4,23174 & 1,19726 & 0,48818\end{array}$

$\begin{array}{lll}-6,52698 & 1,01969 & 2,09501\end{array}$

$\begin{array}{llll}-7,45771 & 0,12947 & 0,37473\end{array}$

$-4,21758 \quad 1,65299 \quad 1,80592$

$-5,35563 \quad 1,56565 \quad 2,60645$

$\begin{array}{lll}-7,41662 & 0,94825 & 2,71054\end{array}$

$-3,30482 \quad 2,08209 \quad 2,19613$

$-5,32227 \quad 1,92643 \quad 3,62863$

$5,48271-0,13307-1,46156$

$6,514690,16212-1,69741$

$5,19728-0,91939-2,16590$

$2,99482-1,30391-0,37554$

$2,69087-0,30606-0,71447$

$3,22891-1,88937-1,28059$

$\begin{array}{llll}-5,48272 & 0,13307 & -1,46156\end{array}$

$-6,51469-0,16212-1,69741$

$\begin{array}{llll}-5,19729 & 0,91939-2,16590\end{array}$

$-2,99482 \quad 1,30388-0,37554$

$-2,690850,30603-0,71445$

$-3,22891 \quad 1,88932-1,28060$

$4,591160,94556-1,74309$

$1,94159-1,928630,33753$

$-4,59117-0,94557-1,74309$

$\begin{array}{llll}-1,94159 & 1,92863 & 0,33752\end{array}$

$4,96969 \quad 2,17694 \quad-1,14925$

$5,94062 \quad 2,50187-1,55429$

$5,06875 \quad 2,07745-0,06166$

$3,90675 \quad 3,22446-1,48809$

$4,30182 \quad 4,21161-1,22059$

$3,76157 \quad 3,22610-2,57380$

$0,76941-2,08807-0,43995$

$0,95342-2,76765-1,28460$

$0,44576-1,12304-0,85363$

$-0,31986-2,65971 \quad 0,47037$

$-0,46108-1,975331,31342$

$0,03715-3,60648 \quad 0,88945$

$-4,96969-2,17695-1,14925$

$-5,06876-2,07745-0,06167$

$-5,94062-2,50188-1,55429$

$-3,90674-3,22446-1,48809$

$-3,76156-3,22610-2,57381$

$-4,30181-4,21161-1,22059$

$-0,76940 \quad 2,08805-0,43995$ 


$\begin{array}{rrrr}\text { H } & -0,44574 & 1,12300 & -0,85359 \\ \mathrm{H} & -0,95341 & 2,76760 & -1,28463 \\ \mathrm{C} & 0,31984 & 2,65974 & 0,47035 \\ \mathrm{H} & 0,46107 & 1,97541 & 1,31343 \\ \mathrm{H} & -0,03718 & 3,60653 & 0,88939 \\ \mathrm{C} & 2,62777 & 3,01580 & -0,81235 \\ \mathrm{C} & 1,58637 & 2,86019 & -0,22844 \\ \mathrm{C} & -1,58638 & -2,86017 & -0,22842 \\ \mathrm{C} & -2,62777 & -3,01579 & -0,81236\end{array}$

\section{3b (meta-monomer)}

$\mathrm{E}=-435281,1961681$ a.u.

$\begin{array}{lrrr}\text { C } & 1,52501 & 1,21497 & 0,24096 \\ \mathrm{C} & 0,94959 & -0,00002 & 0,61496 \\ \mathrm{C} & 1,52496 & -1,21504 & 0,24096 \\ \mathrm{C} & 2,69860 & -1,20746 & -0,51658 \\ \mathrm{C} & 3,28557 & -0,00007 & -0,88904 \\ \mathrm{C} & 2,69866 & 1,20734 & -0,51658 \\ \mathrm{H} & 3,15123 & -2,14558 & -0,82342 \\ \mathrm{H} & 4,19140 & -0,00010 & -1,48548 \\ \mathrm{H} & 3,15132 & 2,14544 & -0,82342 \\ \mathrm{C} & 0,89487 & -2,51845 & 0,70150 \\ \mathrm{C} & 0,89499 & 2,51841 & 0,70150 \\ \mathrm{H} & 1,23445 & 2,75340 & 1,71502 \\ \mathrm{H} & 1,21640 & 3,34504 & 0,05147 \\ \mathrm{O} & -0,52074 & 2,46144 & 0,78798 \\ \mathrm{O} & -0,52085 & -2,46141 & 0,78799 \\ \mathrm{C} & -1,18642 & 2,60339 & -0,45843 \\ \mathrm{H} & -1,22289 & 3,66683 & -0,73797 \\ \mathrm{H} & -0,65973 & 2,05667 & -1,24965 \\ \mathrm{C} & -2,61303 & 2,06155 & -0,31745 \\ \mathrm{H} & -3,20565 & 2,42329 & -1,16603 \\ \mathrm{H} & -3,05564 & 2,49519 & 0,58568 \\ \mathrm{C} & -1,18654 & -2,60334 & -0,45842 \\ \mathrm{C} & -2,61313 & -2,06143 & -0,31745 \\ \mathrm{H} & -3,20577 & -2,42317 & -1,16603 \\ \mathrm{H} & -3,05575 & -2,49504 & 0,58569 \\ \mathrm{C} & -2,66998 & 0,60188 & -0,26739 \\ \mathrm{C} & -2,67001 & -0,60176 & -0,26741 \\ \mathrm{H} & 1,21624 & -3,34509 & 0,05147 \\ \mathrm{H} & 1,23433 & -2,75346 & 1,71502 \\ \mathrm{H} & -1,22306 & -3,66678 & -0,73795 \\ \mathrm{H} & -0,65983 & -2,05665 & -1,24965 \\ \mathrm{H} & 0,02690 & 0,00000 & 1,18080\end{array}$

\section{4b (meta-dimer)}

\begin{tabular}{lrrr}
$\mathrm{E}$ & $=$ & $-870567,3161822$ & \multicolumn{1}{l}{$\mathrm{u}}$. \\
$\mathrm{C}$ & $-5,59212$ & 0,92840 & 0,35778 \\
$\mathrm{C}$ & $-4,24043$ & 0,88637 & 0,71030 \\
$\mathrm{C}$ & $-3,36872$ & 1,91188 & 0,34779 \\
$\mathrm{C}$ & $-3,85800$ & 2,99606 & $-0,38746$ \\
$\mathrm{C}$ & $-5,19982$ & 3,04238 & $-0,74999$ \\
$\mathrm{C}$ & $-6,06486$ & 2,01263 & $-0,38120$ \\
$\mathrm{H}$ & $-3,17822$ & 3,78394 & $-0,68703$ \\
$\mathrm{H}$ & $-5,57321$ & 3,87872 & $-1,33087$ \\
$\mathrm{H}$ & $-7,10861$ & 2,05009 & $-0,67814$ \\
$\mathrm{C}$ & 5,28927 & $-0,97012$ & 0,64048 \\
$\mathrm{C}$ & 6,12408 & $-2,05133$ & 0,34757 \\
$\mathrm{C}$ & 3,99063 & $-0,95994$ & 0,12952 \\
$\mathrm{C}$ & 5,66147 & $-3,10993$ & $-0,42919$ \\
$\mathrm{H}$ & 7,14177 & $-2,06373$ & 0,72593 \\
$\mathrm{C}$ & 3,51895 & $-2,01013$ & $-0,66099$ \\
$\mathrm{C}$ & 4,36239 & $-3,09005$ & $-0,92995$ \\
$\mathrm{H}$ & 6,31580 & $-3,94568$ & $-0,65103$ \\
$\mathrm{H}$ & 4,00223 & $-3,91408$ & $-1,53693$ \\
$\mathrm{C}$ & $-6,52470$ & $-0,18396$ & 0,80177 \\
$\mathrm{H}$ & $-6,84277$ & $-0,01250$ & 1,83450 \\
$\mathrm{H}$ & $-7,42953$ & $-0,18551$ & 0,17607 \\
$\mathrm{C}$ & $-1,92395$ & 1,86178 & 0,78127 \\
$\mathrm{H}$ & $-1,78736$ & 2,45368 & 1,70138 \\
$\mathrm{H}$ & $-1,64093$ & 0,82634 & 1,01832 \\
$\mathrm{C}$ & 5,77352 & 0,15816 & 1,53428 \\
$\mathrm{H}$ & 5,46266 & $-0,03008$ & 2,56628 \\
$\mathrm{H}$ & 6,87330 & 0,19317 & 1,52439 \\
$\mathrm{C}$ & 2,10681 & $-1,98808$ & $-1,18680$ \\
$\mathrm{H}$ & 1,86232 & $-0,99355$ & $-1,58888$ \\
$\mathrm{H}$ & 1,99622 & $-2,71396$ & $-2,00494$ \\
$\mathrm{O}$ & $-5,92073$ & $-1,46867$ & 0,81263 \\
$\mathrm{O}$ & $-1,08944$ & 2,38184 & $-0,24234$ \\
$\mathrm{O}$ & 5,23222 & 1,42952 & 1,21518 \\
$\mathrm{O}$ & 1,21000 & $-2,31186$ & $-0,12775$ \\
$\mathrm{C}$ & $-5,73717$ & $-2,02813$ & $-0,47836$ \\
$\mathrm{H}$ & $-5,20078$ & $-1,33303$ & $-1,13560$ \\
$\mathrm{H}$ & $-6,71630$ & $-2,24463$ & $-0,93207$ \\
$\mathrm{C}$ & $-4,94824$ & $-3,33119$ & $-0,33366$ \\
$\mathrm{H}$ & $-5,45311$ & $-3,95515$ & 0,41184 \\
$\mathrm{H}$ & $-5,00127$ & $-3,87153$ & $-1,28613$ \\
$\mathrm{C}$ & 0,27574 & 2,44236 & 0,13139 \\
$\mathrm{H}$ & 0,66040 & 1,43668 & 0,35371 \\
$\mathrm{H}$ & 0,40518 & 3,05676 & 1,03379 \\
$\mathrm{C}$ & 1,04954 & 3,05662 & $-1,03784$ \\
$\mathrm{H}$ & 0,62916 & 4,04497 & $-1,25193$
\end{tabular}




$\begin{array}{lrrr}\text { H } & 0,87799 & 2,44455 & -1,92951 \\ \mathrm{C} & 5,76316 & 2,00582 & 0,03292 \\ \mathrm{H} & 6,84624 & 2,16465 & 0,14785 \\ \mathrm{H} & 5,60354 & 1,34908 & -0,83101 \\ \mathrm{C} & 5,07625 & 3,35396 & -0,19315 \\ \mathrm{H} & 5,59089 & 3,86857 & -1,01306 \\ \mathrm{H} & 5,21823 & 3,96599 & 0,70416 \\ \mathrm{C} & -0,14016 & -2,39250 & -0,55023 \\ \mathrm{H} & -0,49022 & -1,42219 & -0,93214 \\ \mathrm{H} & -0,24862 & -3,12890 & -1,35969 \\ \mathrm{C} & -0,97620 & -2,82109 & 0,65863 \\ \mathrm{H} & -0,85706 & -2,07428 & 1,45071 \\ \mathrm{H} & -0,56048 & -3,75614 & 1,04849 \\ \mathrm{C} & -3,54757 & -3,14411 & 0,04012 \\ \mathrm{C} & -2,38826 & -2,99554 & 0,32923 \\ \mathrm{C} & 2,47877 & 3,16559 & -0,75974 \\ \mathrm{C} & 3,65103 & 3,24893 & -0,49914 \\ \mathrm{H} & -3,87160 & 0,02391 & 1,25521 \\ \mathrm{H} & 3,34921 & -0,11180 & 0,34237\end{array}$

\section{$3 c$ (para-monomer)}

$\mathrm{E}=-435272,9492007$ a.u.

C $\quad 1,40292-1,55101 \quad 0,07476$

C $\quad 0,75703-1,61133-1,16292$

C $-0,63050-1,60044-1,23711$

C $-1,40291-1,55102-0,07474$

C $-0,75703-1,61128 \quad 1,16294$

C $\quad 0,63051-1,60038 \quad 1,23713$

$\mathrm{H} \quad-1,34466-1,61763 \quad 2,07567$

$\mathrm{H} \quad 1,12026-1,57074 \quad 2,20466$

C $\quad-2,87266-1,22861-0,17735$

C $\quad 2,87267-1,22860 \quad 0,17735$

$\mathrm{H} \quad 3,34596-1,77848 \quad 0,99327$

$\mathrm{H} \quad 3,40063-1,47647-0,75286$

$\begin{array}{llll}0 & 3,04917 & 0,15323 & 0,51141\end{array}$

$\begin{array}{llll}0 & -3,04916 & 0,15322 & -0,51143\end{array}$

C $\quad 2,83762 \quad 1,07759-0,55138$

C $\quad 2,06297 \quad 2,30680 \quad-0,04845$

$\mathrm{H} \quad 2,31242 \quad 3,15062-0,70383$

$\mathrm{H} \quad 2,45332 \quad 2,56008 \quad 0,94310$

C $\quad-2,83765 \quad 1,07759 \quad 0,55135$

C $\quad-2,06297 \quad 2,30680 \quad 0,04843$

$\mathrm{H} \quad-2,31243 \quad 3,15062 \quad 0,70380$

$\mathrm{H} \quad-2,45329 \quad 2,56007 \quad-0,94313$

C $\quad 0,60279 \quad 2,18746-0,00064$

C $-0,60279 \quad 2,18745 \quad 0,00065$

$\begin{array}{rrrr}\mathrm{H} & -3,34595 & -1,77850 & -0,99326 \\ \mathrm{H} & -3,40063 & -1,47647 & 0,75287 \\ \mathrm{H} & -2,29385 & 0,61738 & 1,38113 \\ \mathrm{H} & -3,81675 & 1,40654 & 0,92478 \\ \mathrm{H} & 1,34466 & -1,61772 & -2,07565 \\ \mathrm{H} & -1,12025 & -1,57084 & -2,20464 \\ \mathrm{H} & 2,29380 & 0,61736 & -1,38113 \\ \mathrm{H} & 3,81672 & 1,40652 & -0,92483\end{array}$

\section{4c (para-dimer)}

$\mathrm{E}=-870565,1207541 \mathrm{a} \cdot \mathrm{u}$.

C $\quad 0,57622-3,086890,76255$

C $-0,63096-3,19093 \quad 1,45368$

C $\quad-1,59948-2,19184 \quad 1,36590$

C $-1,34330-1,08092 \quad 0,55410$

C $\quad-0,14331-0,97876-0,13878$

C $\quad 0,83222-1,97761-0,04128$

$\mathrm{H} \quad-2,09386-0,30594 \quad 0,44796$

$\mathrm{H} \quad 0,03345-0,10803-0,76207$

C $\quad-1,16136 \quad 3,08264 \quad 0,33212$

C $-0,81808 \quad 2,78888 \quad 1,65121$

C $\quad-0,14384 \quad 3,13881-0,62572$

C $\quad 0,50952 \quad 2,56403 \quad 2,00791$

$\mathrm{H} \quad-1,59569 \quad 2,71774 \quad 2,40448$

C $\quad 1,18221 \quad 2,91903-0,27211$

C $\quad 1,52354 \quad 2,63170 \quad 1,05292$

$\mathrm{H} \quad 0,75304 \quad 2,31982 \quad 3,03718$

C $\quad 2,12821-1,82647-0,80508$

$\mathrm{H} \quad 1,90505-1,58567-1,85534$

$\mathrm{H} \quad 2,70996-0,98943-0,39897$

C $\quad-2,88919-2,28552 \quad 2,15749$

$\mathrm{H} \quad-3,03186-3,31707 \quad 2,51423$

$\mathrm{H} \quad-2,83772-1,63928 \quad 3,03890$

C $\quad-2,59385 \quad 3,31882-0,06776$

$\mathrm{H} \quad-3,24230 \quad 3,328330,81928$

$\mathrm{H} \quad-2,69177 \quad 4,30008 \quad-0,55937$

C $\quad 2,96303 \quad 2,44218 \quad 1,46463$

$\mathrm{H} \quad 3,38287 \quad 3,40929 \quad 1,78879$

$\mathrm{H} \quad 3,02166 \quad 1,76021 \quad 2,32512$

O $\quad 2,87192-3,03847-0,74185$

$0 \quad-4,04014-1,84047 \quad 1,45271$

$\begin{array}{lllll}0 & -3,00888 & 2,29806 & -0,96938\end{array}$

$\begin{array}{llll}0 & 3,72756 & 1,93717 & 0,38229\end{array}$

C $3,95863-3,15286-1,63884$

$\mathrm{H} \quad 3,75872 \quad-2,60042-2,56617$

$\mathrm{H} \quad 4,03412-4,21574-1,88718$ 


$\begin{array}{rrrr}\text { C } & 5,30708 & -2,71178 & -1,03753 \\ \text { H } & 5,42483 & -3,21765 & -0,07302 \\ \text { H } & 6,10462 & -3,09157 & -1,68913 \\ \text { C } & -4,44216 & -2,70570 & 0,40521 \\ \text { H } & -4,74370 & -3,68094 & 0,81877 \\ \text { H } & -3,62219 & -2,87380 & -0,30333 \\ \text { C } & -5,63594 & -2,07027 & -0,31061 \\ \text { H } & -6,40882 & -1,85578 & 0,43571 \\ \text { H } & -6,05816 & -2,81115 & -0,99991 \\ \text { C } & -4,29307 & 2,54141 & -1,51591 \\ \text { H } & -5,03481 & 2,68065 & -0,71684 \\ \text { H } & -4,27187 & 3,45990 & -2,12334 \\ \text { C } & -4,69268 & 1,35891 & -2,40064 \\ \text { H } & -3,87440 & 1,16822 & -3,10368 \\ \text { H } & -5,55883 & 1,66184 & -3,00121 \\ \text { C } & 5,11913 & 1,94212 & 0,64334 \\ \text { H } & 5,46566 & 2,97395 & 0,80809 \\ \text { H } & 5,34517 & 1,35930 & 1,54734 \\ \text { C } & 5,85313 & 1,34869 & -0,55983 \\ \text { H } & 6,92463 & 1,54470 & -0,43430 \\ \text { H } & 5,53789 & 1,89297 & -1,45677 \\ \text { C } & 5,47974 & -1,27151 & -0,86615 \\ \text { C } & 5,64009 & -0,08505 & -0,73942 \\ \text { C } & -5,30385 & -0,85221 & -1,04638 \\ \text { C } & -5,01569 & 0,14070 & -1,66218 \\ \text { H } & -0,81899 & -4,06793 & 2,06668 \\ \text { H } & -0,39799 & 3,34992 & -1,65901 \\ \text { H } & 1,96359 & 2,95701 & -1,02049 \\ \text { H } & 1,31809 & -3,87136 & 0,83041\end{array}$




\section{Spectra}
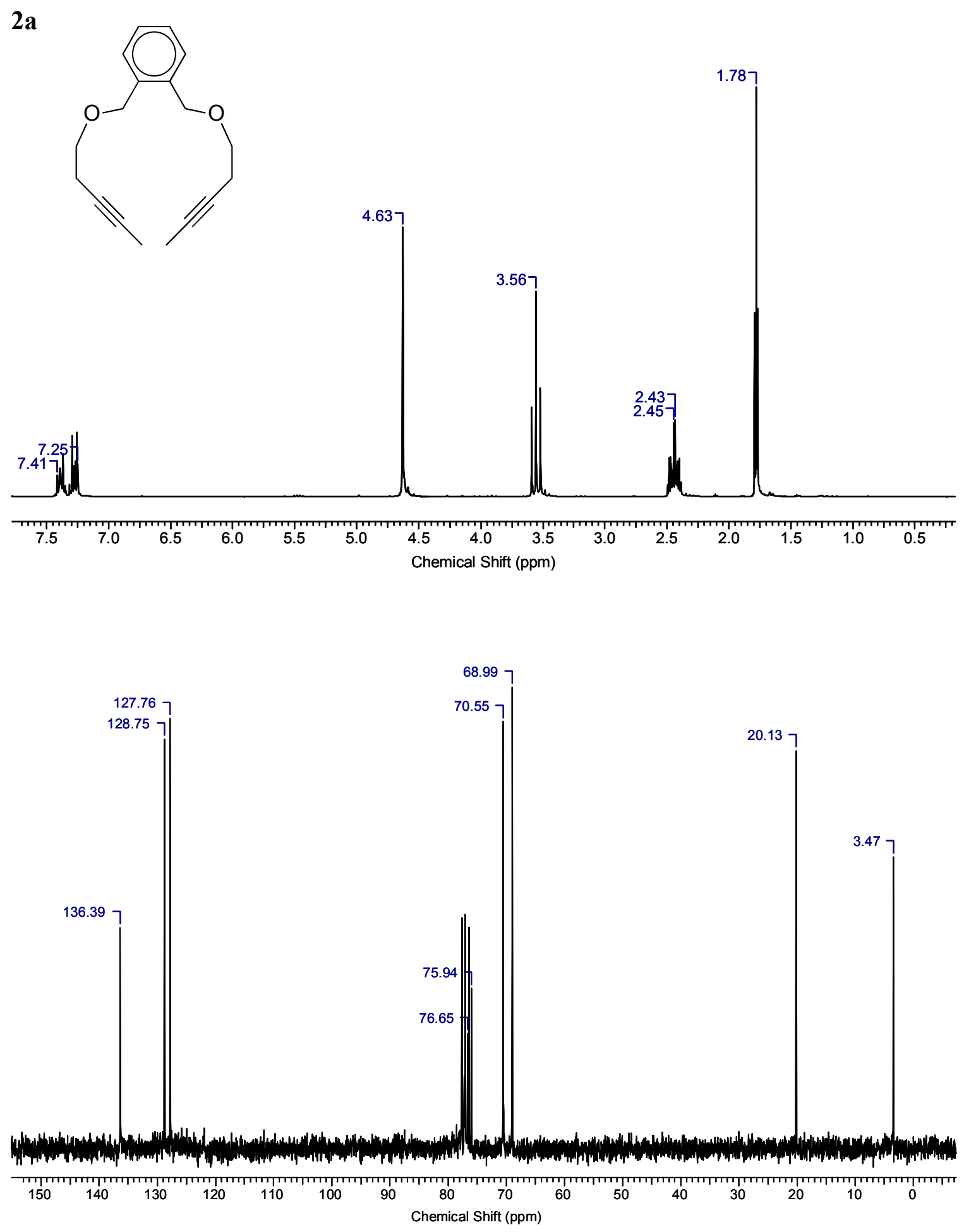

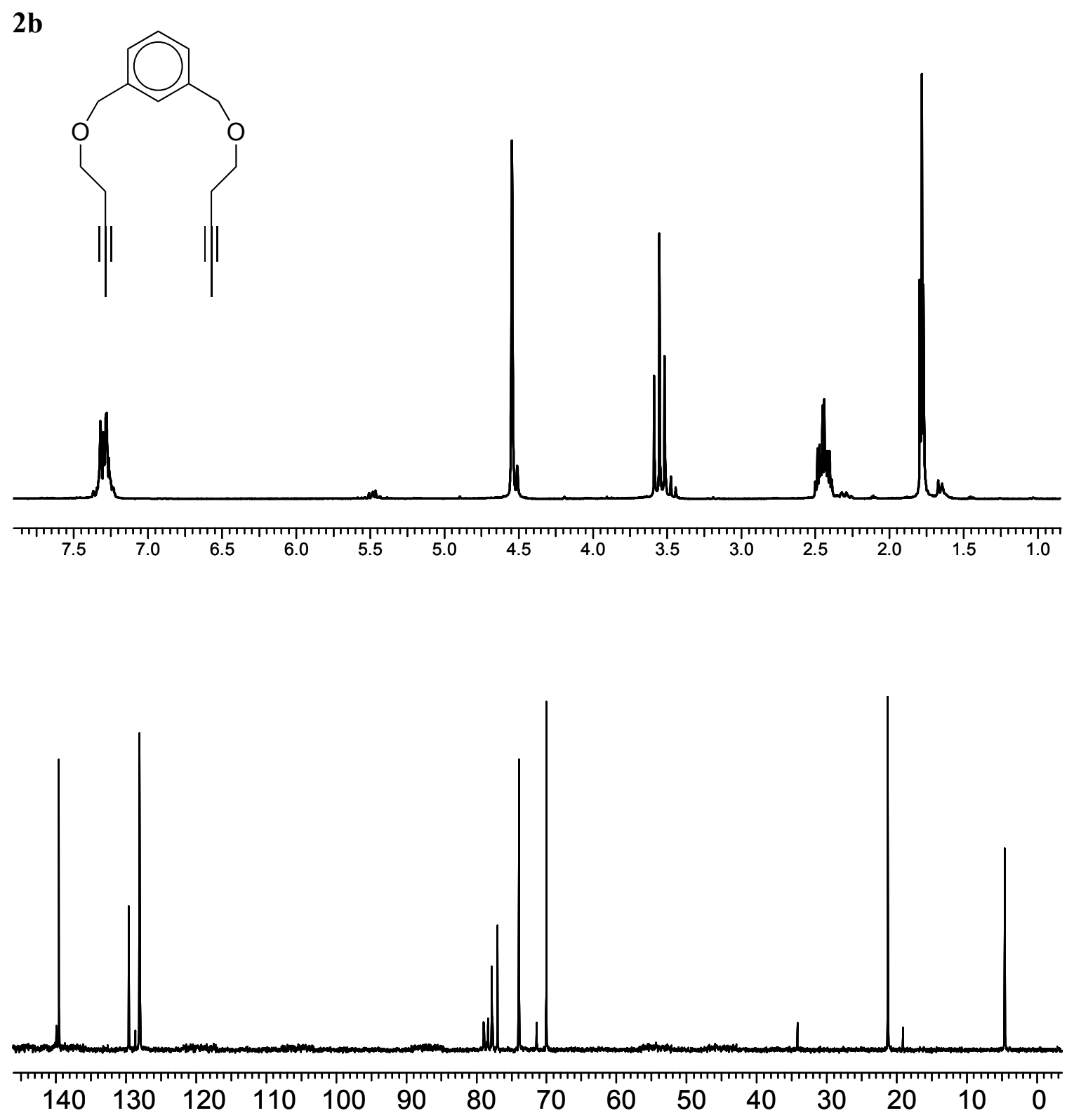
2c
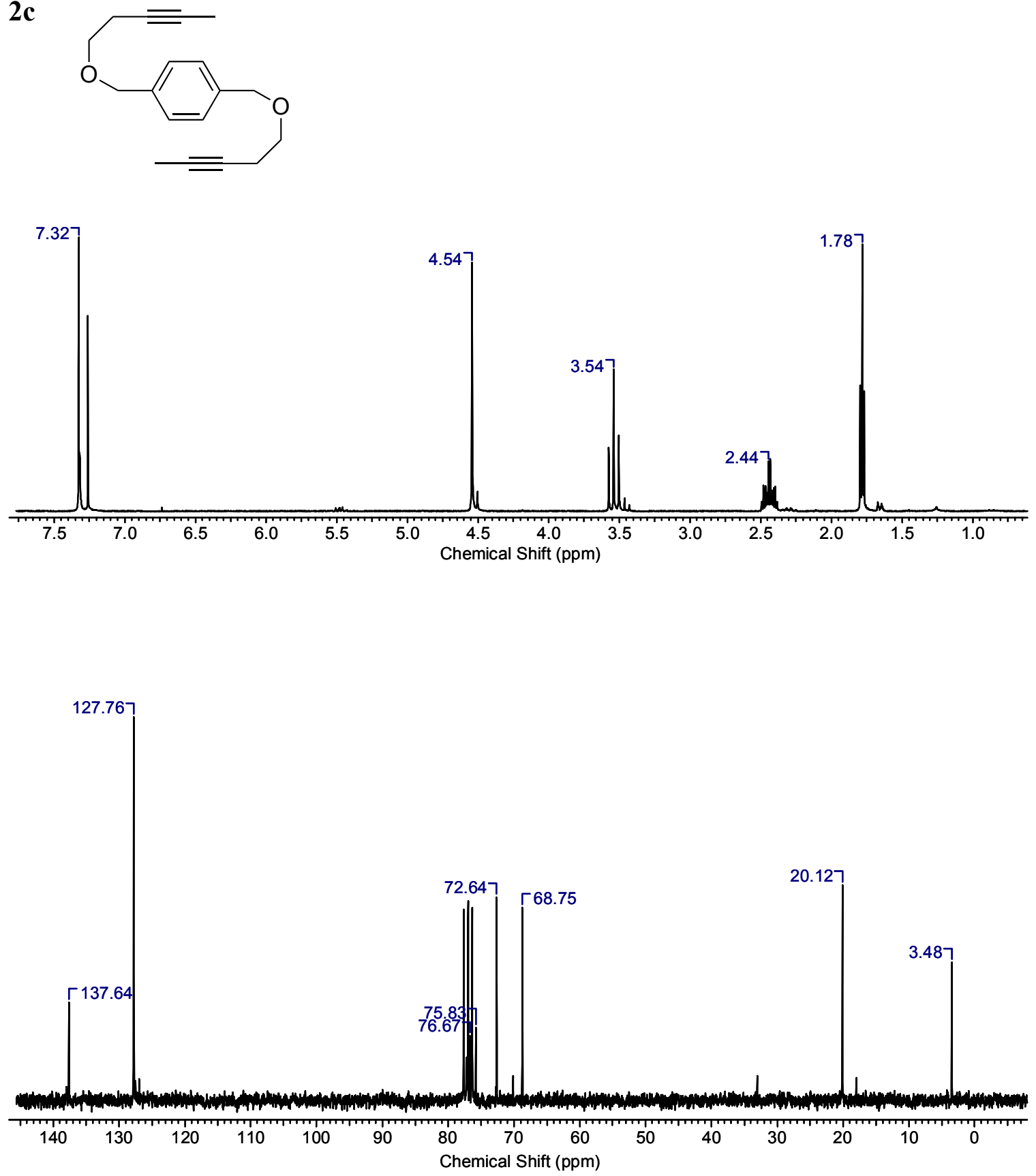


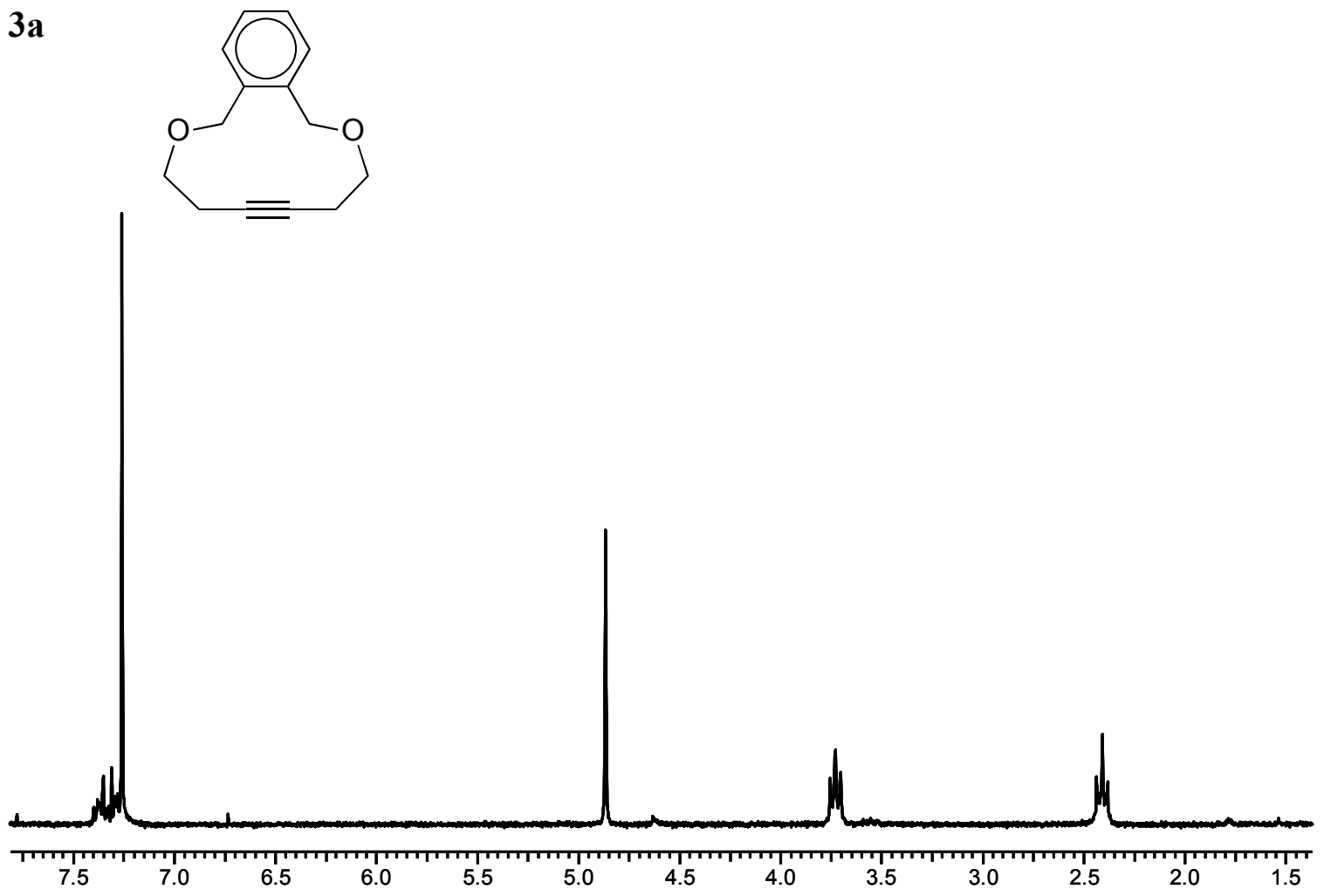

$4 a$

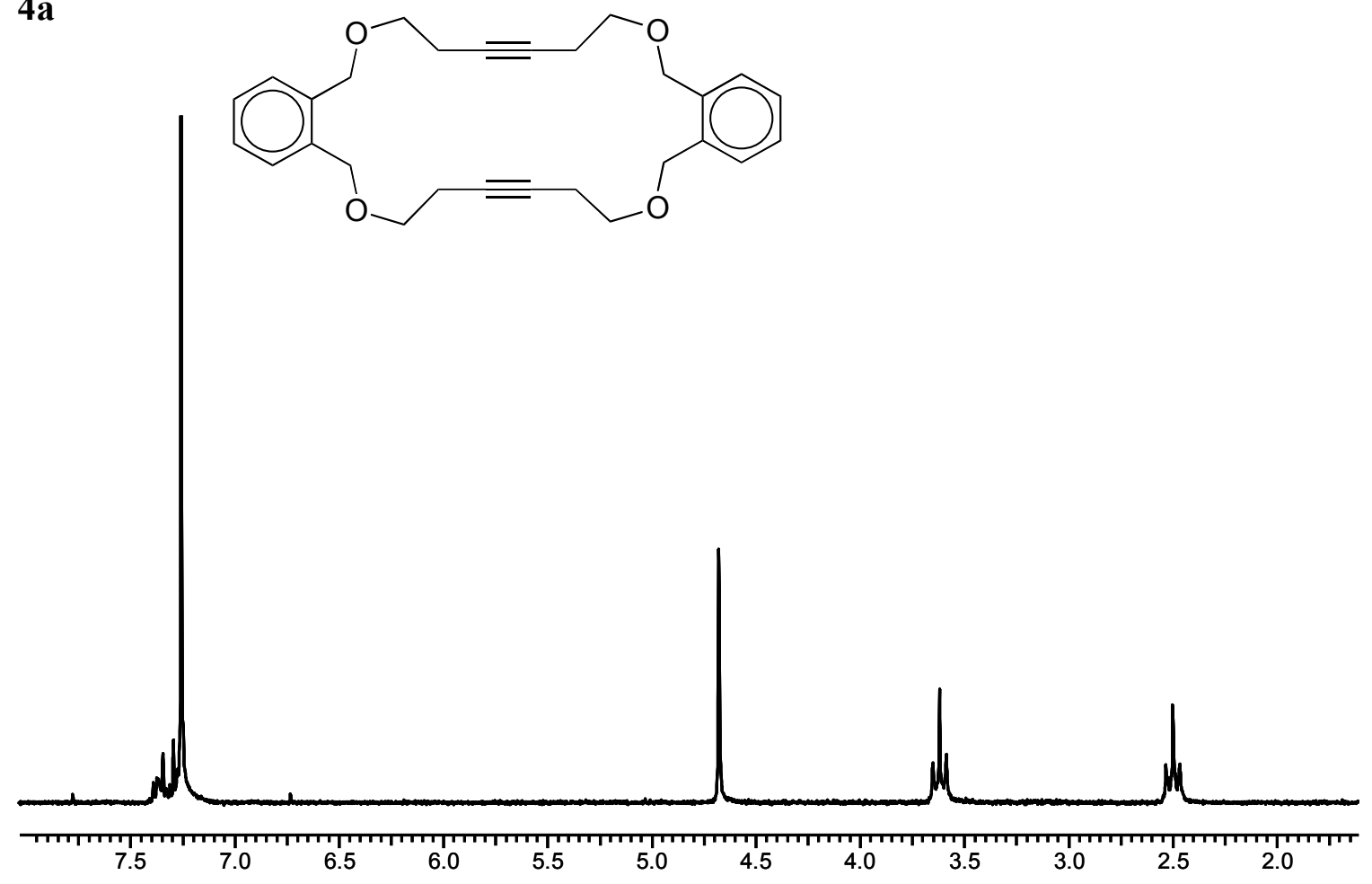

16 
Crude ${ }^{1} \mathrm{H}$ NMR from mixture of $\mathbf{3 a}$ and $\mathbf{4 a}$

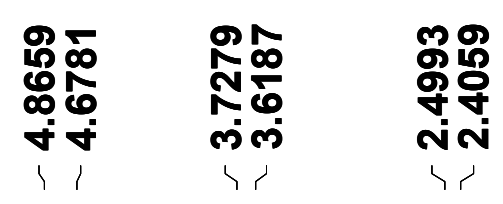

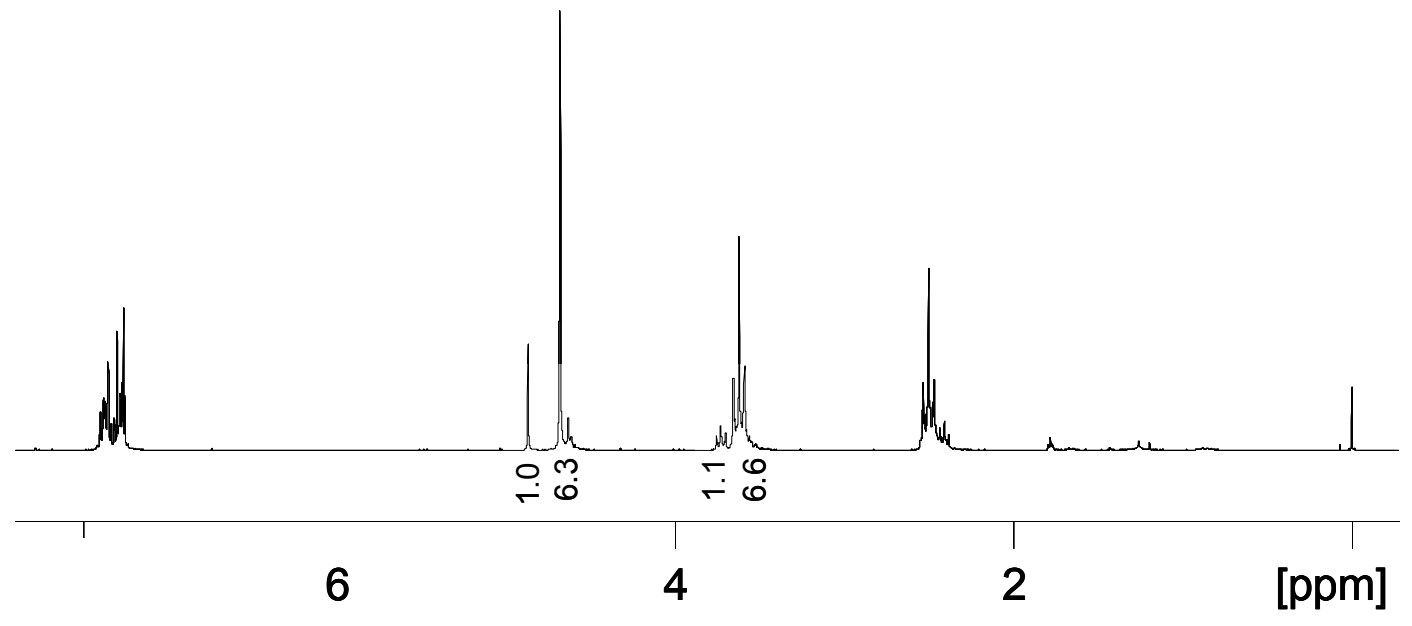



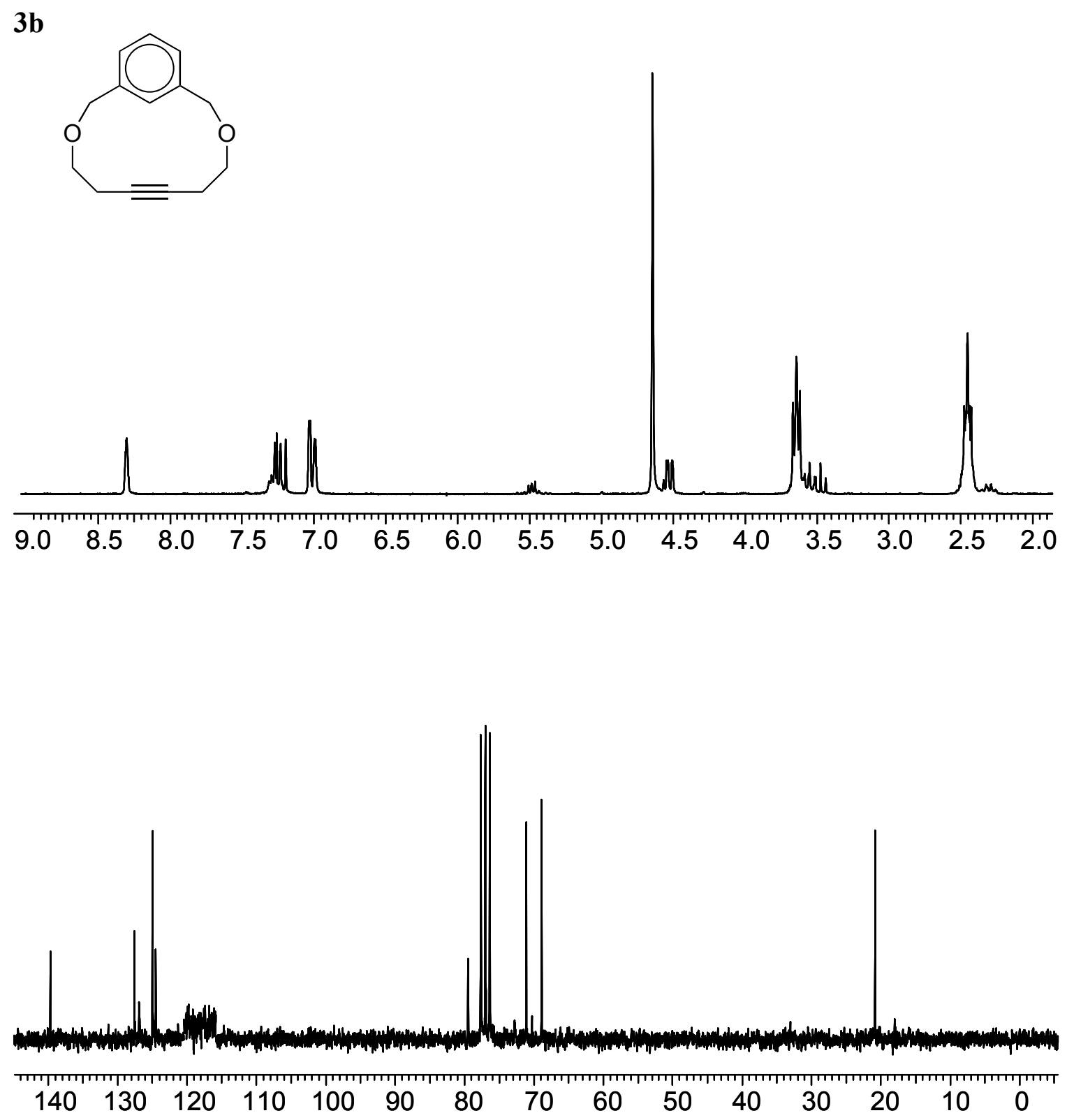

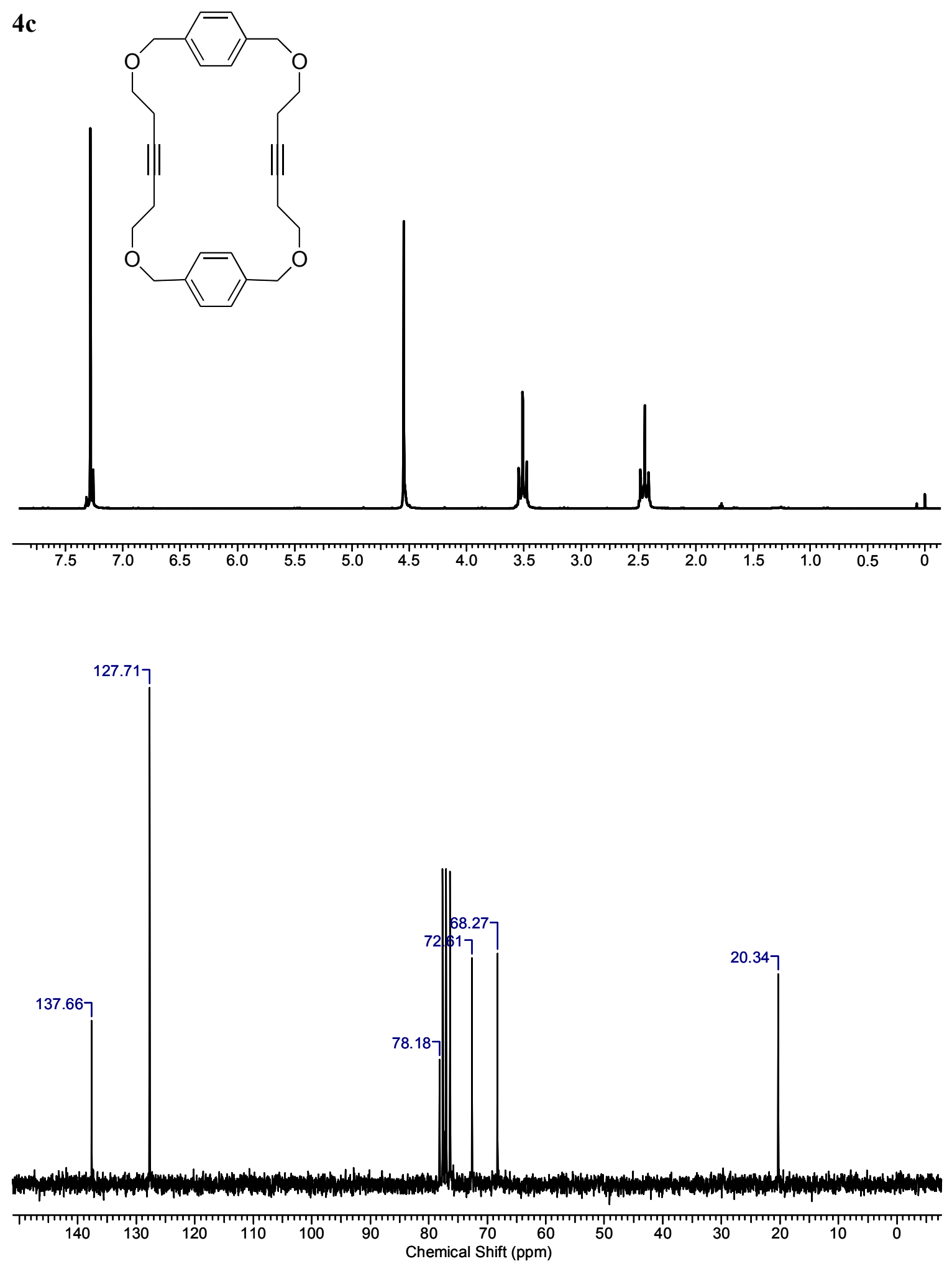